\title{
DESENVOLVIMENTO E AVALIAÇÃO FÍSICO-QUÍMICA DE SORVETE DE MANGA 'TOMMY AKTINS' A BASE DE LEITE DE CABRA
}

Bruno Emanuel Souza Coelho ${ }^{1}$, Erica Antonia Matos de Oliveira ${ }^{1}$, Weslem do Nascimento Guimarães ${ }^{2}$, Ruana de Jesus Santos Correio ${ }^{2}$, Carlos Victor de Castro Miranda ${ }^{1}$, Karla dos Santos Melo de Sousa ${ }^{3}$

\begin{abstract}
RESUMO - O presente artigo teve como objetivo desenvolver três formulações de sorvete de leite de cabra saborizados com diferentes teores de polpa de manga 'Tommy Aktins' (7, 13 e 19\%), e avaliar os atributos físico-químicos da matéria-prima e formulações. As formulações foram avaliadas quanto aos parâmetros físico-químicos de: $\mathrm{pH}$, acidez titulável, sólidos totais, umidade, sólidos solúveis, vitamina $\mathrm{C}$; e cor $\left(\mathrm{L}^{*}, \mathrm{C}^{*} \mathrm{e}\right.$ ${ }^{\circ} \mathrm{H}$ ). As formulações desenvolvidas classificaram-se como um alimento pouco ácido quanto ao $\mathrm{pH}$, teores de sólidos totais e umidade de acordo com a legislação brasileira vigente. As amostras apresentaram baixo teor de Vitamina C. O aumento do percentual de polpa para saborização promoveu saturação de cores, tornando mais brilhante e atrativo o produto, além de caracterizar como produto de coloração amarelada, lembrando a cor da matéria prima utilizada.
\end{abstract}

Palavras chave: caprinocultura, fruticultura, gelados comestiveis, Mangifera indica.

\section{DEVELOPMENT AND EVALUATION PHYSICAL-CHEMICAL OF ICE CREAM OF MANGO 'TOMMY AKTINS' FLAVORED GOAT'S MILK GOAT BASE}

\begin{abstract}
The aim of this paper was to develop three goat milk ice cream formulations flavored with different levels of 'Tommy Aktins' mango pulp (7, 13 and 19\%), and to evaluate the physicochemical attributes of the raw material and formulations. The formulations were evaluated for the physicochemical parameters of: $\mathrm{pH}$, titratable acidity, total solids, moisture, soluble solids, vitamin $C$; and color $\left(L^{*}, C *\right.$ and $\left.{ }^{\circ} H\right)$. The formulations developed were classified as a low acid food in terms of $\mathrm{pH}$, total solids and moisture content in accordance with current Brazilian legislation. The samples presented low Vitamin C content. The increase in pulp percentage for flavoring promoted color saturation, making the product brighter and more attractive, besides characterizing it as a yellowish color product, remembering the color of the raw material used.
\end{abstract}

Keywords: edible ice cream, fruit growing, goat farming, Mangifera indica.

\footnotetext{
${ }^{1}$ Engenheiro (a) Agrônomo (a), Universidade Federal do Vale do São Francisco, campus Ciências Agrárias. E-mail: souza.coelho.18@, gmail.com; erica_oliver_1@hotmail.com; kictorcastro_@hotmail.com.

${ }^{2}$ Graduando em Engenharia Agronômica, Universidade Federal do Vale do São Francisco, campus Ciências Agrárias. E-mail: weslemguimaraes@gmail.com; sruana95@gmail.com.

${ }^{3}$ Professora Adjunta, Universidade Federal do Vale do São Francisco, campus Ciências Agrárias. E-mail: Karla.smsousa@univasf.edu.br.
} 


\section{INTRODUÇ̃̃O}

No Brasil, em especial no Nordeste, a caprinocultura tem grande importância socioeconômica, como fonte alternativa de alimento, devido a capacidade dos caprinos em se adaptar em condições adversas (Coelho et al., 2018).

O consumo de leite de cabra e de seus derivados tem crescido, devido ao seu alto valor nutritivo e a presença de elementos necessários à nutrição humana (proteínas, lipídios, carboidratos, minerais e vitaminas (Garcia \& Travassos, 2012; Lima et al., 2015). Além de seu valor nutricional, o leite de cabra possui algumas características, como maior digestibilidade e de ser menos alergênico, devido a menores concentrações de $\alpha \mathrm{S} 1$ - caseína, proteína que está relacionada à alergia, e maior concentração da proteína $\alpha 1$-caseína, considerada menos alergênica, além disso, apresenta também, menor tamanho e maior dispersão dos seus glóbulos de gordura (Cruz et al., 2016; Silva et al., 2012).

Nos últimos anos, as propriedades benéficas advindas do leite de cabra ganharam reforço, com as inúmeras pesquisas e desenvolvimento de novos produtos derivados desta matéria-prima, disponibilizando alimentos com características funcionais à saúde humana (EMBRAPA, 2015).

A transformação do leite caprino em derivados, condiciona em aumento da base de consumidores ou maior valor agregado, a gama de opções de produtos derivados desta matéria-prima é vasta é um dos caminhos mais claros para o crescimento da caprinocultura de leite (Bomfim et al., 2013).

Almeida (2016) salienta que a crescente demanda por produtos derivados do leite de cabra, como leite em embalagem longa vida, leite em pó, queijos, iogurtes, achocolatado, gelados comestíveis, e outros, tem levado à melhoria do produto final, pelo aumento da vida de prateleira e facilidade de transporte e armazenamento.

Os sorvetes estão classificados na categoria de gelados comestíveis e podem ser apresentados tanto em forma de massa, como em forma de picolé ou soft, segundo a Associação Brasileira das Indústrias de Sorvete (ABIS, 2017). A adição de polpas de frutas para a produção de sorvete pode eliminar a necessidade de adição de saborizantes e corantes artificiais, mas também apresenta desafios técnicos que são associados com o uso da fruta (Carvalho et al., 2018).

A manga apresenta-se como uma fruta com elevada importância para o agronegócio brasileiro, onde em 2016, o Brasil foi responsável pela produção de 1.002.189 toneladas em uma área de 61.842 hectares (IBGE, 2016). A cv. Tommy Atkins caracteriza-se por apresentar frutos grandes, coloração avermelhada, polpa de textura firme, poucas fibras, e sabor agradável (Araújo et al., 2017).

A manga é comercializada quase exclusivamente na forma in natura, mas também pode ser industrializada na forma de suco integral e polpa congelada (Souza, 2015), sendo que a vida útil desse fruto é curta, e apesar da produtividade, e destaque mundial, o Brasil apresenta uma das maiores taxas de perdas pós-colheita de manga, em torno de 10 a 40\% do total produzido (Andrade, 2013), o que justifica o uso de processos para conservar o produto por maior tempo.

Diante do exposto, o objetivo do presente estudo foi desenvolver três formulações de sorvete de leite de cabra saborizados com polpa de manga 'Tommy Aktins', e avaliar os atributos físico-químicos da matéria-prima e das formulações.

\section{MATERIAL E MÉTODOS}

\section{Matéria-prima}

A matéria-prima utilizada para a elaboração do sorvete foi: leite de cabra integral (UHT), açúcar cristal, leite de cabra em pó, emulsificante, liga neutra e gordura vegetal hidrogenada, adquiridos no mercado local do município de Petrolina - PE, e os frutos de manga 'Tommy Atkins' foram adquiridos no mercado local do município de Lagoa Grande - PE.

\section{Obtenção da polpa de manga}

Os frutos de manga foram transportados em caixas isotérmicas até o laboratório, onde foram selecionados manualmente com o objetivo de separar os frutos que apresentaram qualquer tipo de injúria; em seguida, foram lavados em água corrente para retirada de sujidades; sanitizados com solução de hipoclorito de sódio a 100 mg/L de cloro livre, e novamente lavados em água corrente.

A próxima etapa foi o despolpamento: os frutos foram descascados e cortados em pedaços pequenos separando do caroço. O despolpamento foi realizado em liquidificador industrial com capacidade aproximada de 5 litros, os fragmentos foram desintegrados e triturados, e com auxílio de uma peneira de malha de $1,0 \mathrm{~mm}$ foi feito o refinamento da polpa.

As polpas refinadas foram acondicionadas em embalagens de polietileno de baixa densidade, 
com capacidade de aproximadamente $500 \mathrm{~g}$, o produto congelado foi armazenado em freezer horizontal a $-18^{\circ} \mathrm{C}$ até o momento da utilização nos experimentos.

Formulações e ingredientes utilizados para o processamento dos sorvetes

Foram produzidas três formulações de sorvetes com adição de diferentes concentrações de polpa de manga 'Tommy Aktins': 7, 13 e 19\% (correspondendo ao teor de polpa de manga por massa de calda maturada), com quatro repetições. Para a elaboração da base dos sorvetes foram utilizadas as quantidades indicadas na (Tabela 1).

Tabela 1 - Percentual de Formulações dos sorvetes saborizados com polpa de manga 'Tommy Aktins'

\begin{tabular}{lccc}
\hline \multirow{2}{*}{ Ingredientes } & \multicolumn{3}{c}{ Formulações } \\
\cline { 2 - 4 } & $7 \%$ & $13 \%$ & $19 \%$ \\
\hline Leite integral de cabra & 69,93 & 69,93 & 69,93 \\
Açúcar cristal & 6,99 & 6,99 & 6,99 \\
Leite em pó de cabra & 13,99 & 13,99 & 13,99 \\
Gordura vegetal & 6,99 & 6,99 & 6,99 \\
hidrogenada & & & \\
Liga neutra & 1,40 & 1,40 & 1,40 \\
Emulsificante & 0,70 & 0,70 & 0,70 \\
\hline
\end{tabular}

\section{Processamento dos sorvetes}

Para a fabricação dos sorvetes, foi feita uma mistura dos ingredientes secos (açúcar, leite em pó, emulsificante) e adicionados ao leite, posteriormente foi adicionado a gordura vegetal hidrogenada dando origem à calda. Essa calda foi submetida a pasteurização em banho maria á $65{ }^{\circ} \mathrm{C}$ por 30 minutos, e em seguida foi resfriada a temperatura ambiente, para que houvesse a incorporação dos ingredientes a calda foi submetida a maturação em temperatura de congelamento $\left(-18^{\circ} \mathrm{C}\right)$ por 24 horas.

Após a maturação foi feita a adição de emulsificante a calda, em seguida o batimento por meio de agitação constante utilizando uma batedeira doméstica marca Arno Brasil e modelo Facilita SX16, durante 30 minutos para incorporação do ar a mistura.

Posteriormente, em embalagem plástica de propileno estéril com capacidade de 2 litros, o sorvete foi embalado, armazenado, identificado e direcionado ao endurecimento em freezer doméstico vertical, em temperatura média de $-18{ }^{\circ} \mathrm{C}$. As amostras permaneceram no freezer até o momento das análises físico-químicas.

\section{Avaliação físico-química dos sorvetes}

Os parâmetros físico-químicos utilizados na avaliação da polpa de manga foi: $\mathrm{pH}$, sólidos solúveis ( ${ }^{\circ}$ Brix), acidez titulável e vitamina C. Já os parâmetros utilizados para a avaliação formulações dos sorvetes foram: $\mathrm{pH}$, sólidos totais, umidade em base úmida, acidez titulável e vitamina $\mathrm{C}$.

$\mathrm{O}$ pH foi medido em pHmetro digital de bancada calibrado com soluções de tampões valor de $\mathrm{pH}$ 7,0 e 4,0; os sólidos totais e umidade, foram determinados por secagem direta em estufa com circulação forçada de ar a $105^{\circ} \mathrm{C}$ até atingir o peso constante; os sólidos solúveis foi determinado pelo refratômetro do tipo Abbe corrigidos para a temperatura de $20^{\circ} \mathbf{C}$; a acidez titulável foi determinada por volumetria ácido-base empregando a solução de $\mathrm{NaOH}$ a 0,1 Mol.L-1 com o indicador fenolftaleína alcoólica a 1\%, seguindo as metodologias de acordo com o Instituto Adolfo Lutz (IAL, 2008); a vitamina C foi determinada por meio da volumetria de óxido redução, empregando a solução de 2,6-diclorofenol-indofenol a $0,02 \%$ como agente titulante, conforme o método descrito pela AOAC (1997), modificado por Benassi \& Antunes (1998).

Todas as análises foram realizadas em triplicatas.

\section{Avaliação da cor do sorvete}

As formulações de sorvete foram analisadas utilizando um colorímetro da marca Konica-Minolta modelo CR 10. Foram analisados os parâmetros L*, C* e ${ }^{\circ} \mathrm{H}$ que, representam respectivamente, a luminosidade, cromaticidade e ângulo de cor. Todas as leituras foram realizadas em triplicatas.

\section{Análise estatística}

Os dados experimentais obtidos das análises físico-químicas foram analisados estatisticamente através do programa computacional Assistat versão 7.7 Beta (Silva \& Azevedo, 2016), por meio do uso do delineamento inteiramente casualizado (DIC), utilizando o teste de Tukey a nível de 5\% de probabilidade para a comparação entre médias.

\section{RESULTADOS E DISCUSSÃO}

Na Tabela 2, têm-se os valores médios e os desvios padrão dos parâmetros avaliados para a polpa de 
manga variedade Tommy Atkins, utilizada na elaboração das formulações de sorvete. Analisando esta tabela, verifica-se que todos os parâmetros encontram-se de acordo com o Regulamento Técnico para Fixação dos Padrões de Identidade e Qualidade para Polpa de Manga (BRASIL, 2000) que estabelece $\mathrm{pH}$ mínimo de 3,3 e máximo de 4,5 , valor mínimo de $0,32 \mathrm{~g} \cdot 100 \mathrm{~g}^{-1}$ para acidez titulável expressa em ácido cítrico e teor de sólidos solúveis mínimo de $11,00^{\circ}$ Brix.

Tabela 2 - Avaliação físico-química da qualidade da polpa de manga variedade Tommy Atkins utilizada na elaboração do sorvete

\begin{tabular}{lc}
\hline Característica & $\begin{array}{c}\text { Valor } \pm \text { Desvio } \\
\text { padrão }\end{array}$ \\
\hline $\mathrm{pH}$ & $4,07 \pm 0,01$ \\
$\begin{array}{l}\text { Acidez titulável (g de ácido } \\
\left.\text { cítrico. } 100 \mathrm{~g}^{-1}\right)\end{array}$ & $0,412 \pm 0,006$ \\
Sólidos solúveis $\left({ }^{\mathbf{0}} \mathrm{Brix}\right)$ & $17,73 \pm 0,14$ \\
Vitamina C $\left(\mathrm{mg} .100 \mathrm{~g}^{-1}\right)$ & $9,647 \pm 0,621$ \\
\hline
\end{tabular}

Quanto o teor de ácido ascórbico (vitamina C) a legislação brasileira não estabelece um valor mínimo ou máximo. Entretanto, os valores encontrados no presente trabalho foram superior ao descrito pela Tabela Brasileira de Composição Química de Alimentos (TACO), que descreve um teor médio de 7,9 mg. $100 \mathrm{~g}^{-1}$ deste composto para polpa de manga congelada (UNICAMP, 2011).

Conforme os dados expressos na Tabela 3 é possível verificar que o $\mathrm{pH}$ das formulações variou de 6,09 à 6,30 . Nota-se diferença significativas em todos os tratamentos, sendo perceptível a redução desse parâmetro em função do aumento da adição de polpa para a saborização. Ademais, ambas as formulações apresentaram $\mathrm{pH}$ superior ao valor de 5,68 encontrado por de Correia et al. (2008), que avaliou as características químicas e as propriedades de derretimento de sorvete de leite de cabra saborizados com polpa de goiaba.

Segundo Gava, Silva e Gava (2008), é possível classificar o alimento quanto o grau de acidez com o valor do $\mathrm{pH}$, que mede a concentração de $\mathrm{H}+$ de um alimento, em três grupos pouco ácido $(\mathrm{pH}>4,5)$, alimentos ácidos ( $\mathrm{pH} 4-4,5)$, e muito ácidos $(\mathrm{pH}<4,0)$. Com isso ambas as formulações apresentaram valor de $\mathrm{pH}$ superior a 4,5, classificando-se como alimentos pouco ácido.

Tabela 3 - Avaliação físico-química do sorvete de leite de cabra saborizado com polpa de manga da variedade Tommy Atkins

\begin{tabular}{|c|c|c|c|c|}
\hline \multirow{2}{*}{ Parâmetro } & \multicolumn{3}{|c|}{ Porcentagem de polpa de manga } & \multirow{2}{*}{$\mathrm{CV}(\%)$} \\
\hline & $7 \%$ & $13 \%$ & $19 \%$ & \\
\hline $\mathrm{pH} *$ & $6,30 \pm 0,01 \mathrm{a}$ & $6,16 \pm 0,01 b$ & $6,09 \pm 0,01 \mathrm{c}$ & 0,20 \\
\hline $\begin{array}{l}\text { Acidez titulável } \\
\left(\mathrm{g} \text { de ácido cítrico. } 100 \mathrm{~g}^{-1}\right) *\end{array}$ & $0,178 \pm 0,005 \mathrm{~b}$ & $0,188 \pm 0,001 \mathrm{a}$ & $0,196 \pm 0,003 \mathrm{a}$ & 1,81 \\
\hline Sólidos totais $(\%) *$ & $32,89 \pm 0,19 \mathrm{a}$ & $31,80 \pm 0,10 b$ & $31,61 \pm 0,23 \mathrm{~b}$ & 0,57 \\
\hline Umidade $(\% . u b u) *$ & $67,11 \pm 0,19 b$ & $68,20 \pm 0,10 b$ & $68,39 \pm 0,23 \mathrm{a}$ & 0,27 \\
\hline Sólidos solúveis $\left({ }^{\circ} \mathrm{Brix}\right)^{\mathrm{ns}}$ & $28,10 \pm 0,68 \mathrm{a}$ & $27,93 \pm 0,28 \mathrm{a}$ & $27,84 \pm 0,08 \mathrm{a}$ & 1,55 \\
\hline Ácido ascórbico $\left(\mathrm{mg} \cdot 100 \mathrm{~g}^{-1}\right)^{\mathrm{ns}}$ & $1,106 \pm 0,045 \mathrm{a}$ & $1,105 \pm 0,052 \mathrm{a}$ & $1,116 \pm 0,035 \mathrm{a}$ & 4,06 \\
\hline
\end{tabular}

Médias seguidas da mesma letra na mesma linha não diferem estatisticamente pelo modelo de Tukey. * significativo ao nível de 5\% de probabilidade; ${ }^{\text {ns }}$ não significativo; $\mathrm{CV}$ - coeficiente de variação.

A acidez titulável dos gelados comestíveis a base de leite é um parâmetro influenciado pela fruta utilizada na saborização. Tendo em vista que a polpa de manga utilizada como matéria-prima apresentou teor considerável de acidez, os sorvetes expressaram coerência com este dado, pois com o aumento da adição da polpa foi constatado aumento significativo deste ácido em todas as formulações. De acordo com Lamounier et al. (2015), este fato pode ser justificado devido a incorporação de ingredientes com menor acidez ao leite, principalmente polpa de frutas. 
Todas as formulações desenvolvidas apresentaram teores de sólidos totais acima do valor de $26 \%$, que é considerando o teor mínimo para gelados comestíveis a base de leite (BRASIL, 2005a). Estes sólidos são desejados pelos consumidores, pois atuam melhorando a textura e a cremosidade do produto elaborado, entretanto, constatou-se que maiores teores de polpa reduziram significativamente o teor destes sólidos, isso justifica-se ao elevado teor de água presente na polpa da manga 'Tommy Aktins'.

Em relação a umidade, a formulação em que foi elaborada com maior teor de polpa para a saborização (19\%), apresentou uma maior umidade. Paula et al. (2010) obtiveram valores inferiores ao do presente trabalho para três formulações de sorvete de leite de cabra adoçado com mel de abelhas africanizadas, variando de 60,78 a $64,19 \%$. No presente trabalho os valores foram superiores, justificando-se ao elevado teor de água presente na polpa de manga.

Em relação à análise de sólidos solúveis ( $\left.{ }^{\circ} \mathrm{Brix}\right)$, os resultados obtidos não apresentaram diferença significativa para as diferentes formulações desenvolvidas (Tabela 3), e inferiores ao relatado por Lamounier et al. (2015), que foi de 34 a $36^{\circ}$ Brix ao avaliar três formulações de sorvetes enriquecidos com farinha de jabuticaba. Segundo Perrone et al. (2011), um elevado teor de Brix pode influenciar na aceitação do produto, pois está diretamente relacionado com o sabor.

Quanto ao parâmetro de vitamina C (ácido ascórbico), os sorvetes apresentam valores médios entre
1,10 e $1,11 \mathrm{mg} \cdot 100 \mathrm{~g}^{-1}$. Não foram observados os teores consideráveis contido na matéria-prima nas formulações de sorvete, e isso pode ser justificado pelo fato de o teor de ácido ascórbico, ser rapidamente oxidado quando exposto ao ar, tornando-se mais sensível à degradação durante o processamento e estocagem, e por possuir características de uma vitamina hidrossolúvel e termolábil (Araújo et al., 2009; Dantas et al., 2010).

E sabendo que sua Ingestão Diária Recomendada (IDR) de Vitamina C para adultos é de $45 \mathrm{mg}$ (BRASIL, 2005b). As formulações desenvolvidas apresentam baixo teor diante dessa quantidade requerida pelo organismo de um adulto, entretanto pode ser considerada como uma fonte complementar.

Os parâmetros de cor (Tabela 4) revelaram diferença significativa de luminosidade $\left(\mathrm{L}^{*}\right)$, atestando menores valores para o sorvetes saborizado com maiores percentuais de polpa. Sendo assim, de acordo com Prati et al. (2005), isso indica que o aumento da concentração de polpa, resultará em um produto mais escuro, visto que o leite é opaco e claro.

Em relação a cromaticidade (croma) das formulações, observa-se que as diferentes proporções de polpa adicionada para saborização influenciaram de forma significativa. Constata-se que, houve uma saturação de cores com o aumento da adição de polpa. Segundo Pathare et al. (2013), isso resulta em um produto mais brilhante e mais atrativo sensorialmente.

Tabela 4 - Avaliação colorimétrica do sorvete de leite de cabra saborizado com polpa de manga da variedade Tommy Atkins

\begin{tabular}{lcccc}
\hline Característica & \multicolumn{3}{c}{ Porcentagem de polpa de manga } & CV (\%) \\
\cline { 2 - 4 } & $7 \%$ & $13 \%$ & $19 \%$ & 2,99 \\
\hline Luminosidade $\left(\mathrm{L}^{*}\right)^{*}$ & $69,74 \pm 0,68 \mathrm{a}$ & $66,78 \pm 0,08 \mathrm{a}$ & $60,35 \pm 0,54 \mathrm{~b}$ & 0,34 \\
Croma $\left(\mathrm{C}^{*}\right)^{*}$ & $14,04 \pm 2,76 \mathrm{c}$ & $18,23 \pm 1,80 \mathrm{~b}$ & $26,25 \pm 0,10 \mathrm{a}$ & 0,34 \\
Ângulo de cor $\left({ }^{\circ} \mathrm{H}\right)^{*}$ & $100,22 \pm 1,85 \mathrm{a}$ & $97,43 \pm 0,58 \mathrm{~b}$ & $95,28 \pm 0,16 \mathrm{c}$ & 0,34 \\
\hline
\end{tabular}

Médias seguidas da mesma letra na mesma linha não diferem estatisticamente pelo modelo de Tukey. * significativo ao nível de $5 \%$ de probabilidade; ${ }^{\text {ns }}$ não significativo; $\mathrm{CV}$ - coeficiente de variação.

Ao aumentar a concentração de polpa, verificase uma redução da intensidade de verde e aumento da intensidade da intensidade de amarelo, evidenciado pela redução do ângulo hue $\left(\mathrm{H}^{\circ}\right)$, que depende da quantidade relativa de cores vermelha e amarela. Entretanto, todos os valores estão dentro do primeiro quadrante e próximos ao ângulo de $90^{\circ}$ da escala de HunterLab, citado por Lanozo
(1978), caracterizando um produto de forte coloração amarela, característica da matéria-prima utilizada.

Os resultados encontrados foram próximos ao relatado por Santos et al. (2016), ao avaliarem as características físico-químicas do iogurte liofilizado e reidratado, que constataram valor maior para o iogurte reidratado, devido à concentração dos grânulos de açúcar 
que originam uma cromaticidade de 9,30 para o in natura e 11,83 para o reidratado, da mesma forma aconteceu com os valores de ${ }^{\circ} \mathrm{H}$, visto que as amostras apresentaram valores de $89,13^{\circ}$ e $87,30^{\circ}$ para o in natura e reidratado respectivamente, indicando que as amostras estão evoluindo para o amarelo.

\section{CONCLUSÕES}

Ao aumentar o teor de polpa de manga 'Tommy Aktins' na saborização do sorvete, evidenciou-se aumento significativo da acidez titulável no produto final.

Todas as formulações desenvolvidas apresentaram teores de sólidos totais e umidade de acordo com a Legislação Brasileira vigente.

Nota-se que as formulações desenvolvidas apresentaram baixo teor de vitamina $\mathrm{C}$, caracterizando-se como fonte complementar.

Maiores teores de polpa adicionados para a saborização proporcionou menores valores de $\mathrm{L}^{*}$, indicado escurecimento.

O aumento do percentual de polpa para saborização promoveu saturação de cores, tornando o produto mais brilhante e atrativo sensorialmente.

\section{LITERATURA CITADA}

ABIS. Associação Brasileira das indústrias e do setor de sorvete. Disponível em: <http://www.abis.com.br/> Acesso em 02 abr. 2017.

ALMEIDA, A. B. S.; FERREIRA, M. A. C.; BARBOSA, T. A.; SIQUEIRA, A. P. S.; SOUZA, E. R. B. Elaboração e avaliação sensorial de sorvete diet e sem lactose de mangaba endêmica do Cerrado. Revista de Agricultura Neotropical, v. 3, p. 38-41, 2016.

ANDRADE, M. E. L. Qualidade pós-colheita de manga 'Tommy Atkins' tratada com água eletrolisada. 2013. 66p. Tese (Doutorado em Fitotecnia. Área de concentração: Agricultura Tropical) - Universidade Federal Rural do SemiÁrido, Mossoró- RN, 2013.

ARAÚJO, D. O.; MORAES, J. A. A.; CARVALHO, J. L. M. Fatores determinantes na mudança do padrão de produção e consumo da manga no mercado nacional. Revista em Agronegócio e Meio Ambiente, v. 10, Edição Especial, p. 5173, 2017.

ARAUJO, E. R.; ALVES, L. I. F.; RÊGO, E. R.; RÊGO, M. M.; CASTRO, J. P.; SAPUCAY, M. J. L. C. Caracterização físico-química de frutos de biri-biri (Averrhoa bilimbi L.). Revista Biotemas, v. 22, n. 4, 2009.

ASSOCIATION OF OFFICIALANALYTICALCHEMISTS. Official methods of analysis of AOAC international. 16th ed. Gaithersburg: AOAC international, 1997. 1141p.

BENASSI, M. T.; ANTUNES, A. J. A. Comparisson of meta-phosphoric and oxalic acids as extractant solutions for determination of vitamin $\mathrm{C}$ in selected vegetables. Arquivos de Biologia e Tecnologia, v.31, n. 4, p. 507-513, 1998.

BOMFIM, M. A. D. et al. Produção e qualidade do leite de cabra no Brasil. In. XXIII Congresso Brasileiro de Zootecnia. Universidade Estadual do Oeste do Paraná. Foz do Iguaçu, 2013.

BRASIL, Ministério da Saúde. Agência Nacional de Vigilância Sanitária (ANVISA). Resolução RDC n.269, de 22 de setembro de 2005. Regulamento técnico sobre a ingestão diária recomendada (IDR) de proteína, vitaminas e minerais. Diário Oficial da República Federativa do Brasil, Brasília, 23 de setembro de 2005 b.

BRASIL. Ministério da Agricultura, Abastecimento e Pecuária. Instrução Normativa ${ }^{\circ}$ 01, de 07 de janeiro de 2000. Aprova o regulamento técnico geral para fixação dos padrões de identidade e qualidade para polpa de fruta. Diário Oficial [da] União. Brasília, DF, 10 jan. 2000. Seção 1, nº 06. p.54.

BRASIL. Secretaria de Vigilância Sanitária. Resolução RDC $\mathrm{n}^{\mathrm{o}} 266$, de 22 de setembro de 2005. Aprova o regulamento gelados comestiveis e preparados para gelados comestiveis. Diário Oficial da União, Poder Executivo, 23 de set. 2005a.

CARVAlHO, V. S.; ASQUIERI, E. R.; DAMIANI, C. Produção de sorvete utilizando a polpa de sapota (Quararibea cordata vischer). Revista Agrarian, v.11, n.40, p. 189-194, 2018.

COELHO, M. C. S. C.; RODRIGUES, B. R.; COELHO, M. I. S.; LIBÓRIO, R. C.; COSTA, F. F. P.; SILVA, G. L. S. Características físico-química e microbiológica do leite de cabra produzido em Petrolina-PE. Agropecuária Científica do Semiárido, v.14, n.3, p.175-182, 2018.

CORREIA, R. T. P.; MAGALHÃES, M. M. A.; PEDRINI, M. R. S.; CRUZ, A. V. F.; CLEMENTINO, I. M. Sorvetes elaborados com leite caprino e bovino: composição química e propriedades de derretimento. Revista Ciência Agronômica, v. 39, p. 251-256, 2008.

CRUZ, A. G. D.; OLIVEIRA, C. A. F.; ZACARCHENCO, P. B.; CORASSIN, C. H. Química, Bioquímica, Análise Sensorial e Nutrição no Processamento de Leite e Derivado. 1. ed, Rio de Janeiro: Elsevier, 2016. 
DANTAS, R. L.; ROCHA, A. P. T.; ARAÚJO, A. S.; RODRIGUES, M. S. A.; MARANHÃO, T. K. L. Perfil da qualidade de polpas de fruta comercializadas na cidade de Campina Grande/PB. Revista verde. Mossoró, v. 5, n. 5, p. 6166, 2010.

EMBRAPA. Leite de cabra funcional oferece vantagens adicionais para a saúde. Disponível: https://www.embrapa. br/. Acesso: 06 de outubro de 2015.

GARCIA, R. V.; TRAVASSOS, A. E. R. Aspectos gerais sobre o leite de cabra: uma revisão. Revista do Instituto de Laticínios Cândido Tostes, v. 67, n. 386, p. 81-88, 2012.

GAVA, A. J.; SILVA, C. A. B.; GAVA, J. R. F. Tecnologia de alimentos: princípios e aplicações. São Paulo: Nobel, 2008. $511 \mathrm{p}$.

GUIMARÃES, V. P. Curva de lactação, efeitos ambientais e genéticos sobre o desempenho produtivo de cabras leiteiras. 2004. 87 f. Dissertação (Mestrado em Zootecnia) Universidade Federal de Viçosa, Viçosa, 2004.

IBGE. Banco de dados agregados do Instituto Brasileiro de Geografia e Estatística. 2016. <http://www.sidra.ibge.gov. br>. Acesso em: 15 jun. 2018.

INSTITUTO ADOLFO LUTZ. Normas analiticas do Instituto Adolfo Lutz: métodos químicos e fisicos para análises de alimentos. $4^{\mathrm{a}}$ Ed., 1a ed. Digital, São Paulo, 2008. 1020p.

LAMOUNIER, M. L.; ANDRADE, F. C.; MENDONÇA, C. D.; MAGALHÃES, M. L. Desenvolvimento e caracterização de diferentes formulações de sorvetes enriquecidos com farinha da casca da jabuticaba (Myrciaria cauliflora). Revista do Instituto de Laticínios Cândido Tostes, v. 70, p. 93, 2015.

LOZANO, R. D. El color y su medición, Ed. I Américalee S.R.L, Buenos Aires, 1978. 640p.

LIMA, F. T.; STURN, R. G.; TAVOLARO, P.; RIBEIRO, A. R. B.; SOUSA, V. A. F. Estudo exploratório do mercado das potencialidades de consumo do leite de cabra e seus derivados entre paulistanos. Informações Econômicas, v. 45, n. 3, 2015.

MARQUES, A. P. S. M.; CHICAYBAM, G.; ARAUJO, M. T.; MANHÃES, L. R. T.; SABAA-SRUR, A. U. O. Composição centesimal e de minerais de casca e de polpa de manga (Mangifera indica L.) cv. Tommy Atkins. Revista Brasileira de Fruticultura (Impresso), v. 32, p. 1206-1210, 2010.
PATHARE, P. B.; OPARA, U. L.; AL-SAID, F. A. Colour measurement and analysis in fresh and processed foods: a review. Food and Bioprocess Technology, v. 6, p. 36-60, 2013.

PAUlA, C. M.; PORTELA, M. C. C.; PAUlA, J. A.; PEREIRA, J. O. P.; SANTOS, K. M. O. Sorvete potencialmente probiótico de leite de cabras, sabor morango, adoçado com açúcar e mel de abelhas africanizadas. In: MOTA, A. S. M. F. Coletânea BITEC 2008-2010. 8. ed. Brasília, 2010, p.89-102.

PERRONE, I. T.; RENHE, I. R. T.; PEREIRA, J. P. F.; COlOMBO, M.; COElHO, J. S.; MAGAlHÃES, F. A. R. Influência de diferentes espessantes nas características sensoriais do doce de leite para confeitaria. Revista do Instituto de Laticínios Cândido Tostes, v. 66, n. 379, p. 45-50, 2011.

PRATI, P.; MORETTI, R. H.; CARDELLO, A. B. Elaboração de bebida composta por mistura de garapa parcialmente clarificada - estabilizada e sucos de frutas ácidas. Revista Ciência e Tecnologia de Alimentos, v. 25, n. 1, p. 147-152, 2005.

SANTOS, G.; CUNHA, V. C. M.; PEDRAL, A. L.; SILVA, G. F.; SILVA, M. A. A. P.; CASTRO, A. A. Avaliação das características físico-químicas do iogurte liofilizado e reidratado. Proceeding of ISTI/SIMTEC, v. 3, p. 723-728, 2016.

SILVA, F. A. Z.; AZEVEDO, C. A. V. The assistat software version 7.7 and its use in the analysis of experimental data. African Journal of Agricultural Research, v.11, n.39, p. 37333740, 2016.

SILVA, H. W.; GUIMARÃES, C. R.; OLIVEIRA, T. S. Aspectos da exploração da caprinocultura leiteira no Brasil. Revista Brasileira de Agropecuária Sustentável, v. 2, n. 2, p. 121-125, 2012.

SOUZA, M. E. A. O. Potencial antioxidante de extratos da casca de manga (Mangifera indica L.) da variedade Tommy Atkins obtidos por métodos a baixa e a alta pressão e dimensionamento de uma coluna para extração supercrítica. 2015. 191 f. Tese de doutorado (Programa de Pós-Graduação em Engenharia de Alimentos) - Universidade Federal de Santa Catarina (UFSC), Florianópolis-SC, 2015.

UNICAMP. Tabela brasileira de composição de alimentos (TACO)/NEPA - UNICAMP. 4a ed. rev. e ampl. Campinas: NEPA- UNICAMP, 2011. 161p. 